\title{
Venous Thromboembolism and Risk of Cancer in Patients with Diverticular Disease: A Danish Population-Based Cohort Study
}

\section{Louise Thomsen (iD* \\ Frederikke Schønfeldt \\ Troelsen (1D* \\ David Nagy (D) \\ Nils Skajaa (D) \\ Dóra Körmendiné Farkas (DD \\ Rune Erichsen (D)}

Department of Clinical Epidemiology, Aarhus University Hospital, Aarhus N, 8200, Denmark

*These authors contributed equally to this work

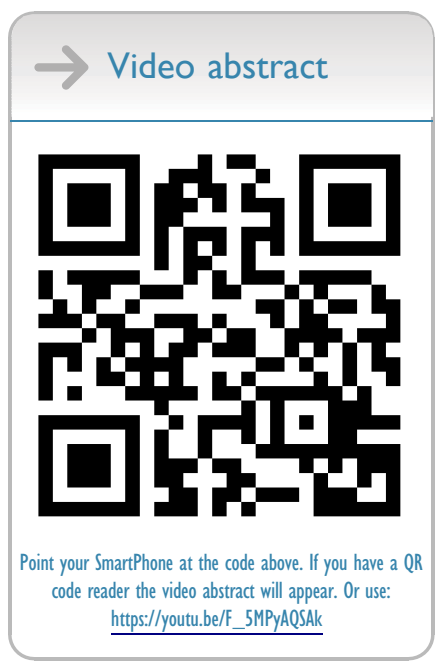

Correspondence: Louise Thomsen Department of Clinical Epidemiology, Aarhus University Hospital, Olof Palmes Allé 43-45, Aarhus N, 8200, Denmark Tel +4520477642

Email louisethomsen3@gmail.com
Purpose: Venous thromboembolism may be a harbinger of cancer. Patients with diverticular disease are suggested to have an increased risk of developing venous thromboembolism compared with the general population, but it remains unclear whether venous thromboembolism is also a marker of occult cancer in these patients. We investigated the risk of cancer after venous thromboembolism among patients with diverticular disease.

Patients and Methods: We used Danish health registries to conduct a nationwide, population-based cohort study during 1996-2017. We identified all venous thromboembolism patients with a diagnosis of diverticular disease and calculated absolute risks of cancer and standardized incidence ratios (SIRs) by comparing observed and expected cancer incidence based on national cancer incidence in the Danish population.

Results: We followed 3406 patients with venous thromboembolism and diverticular disease for a median of 3.0 years (interquartile range: 1.0-6.0). During the first year of follow-up, we observed 212 cancer cases. The corresponding one-year risk of cancer was $6.2 \%$ (95\% confidence interval [CI]: 5.5-7.1) with a SIR of 2.9 (95\% CI: 2.5-3.3). The SIRs were particularly elevated for cancers of the stomach, pancreas, ovary, and kidney. During the second and subsequent years of follow-up, 337 cancers were diagnosed with a SIR of 1.1 (95\% CI: 1.0-1.3).

Conclusion: Venous thromboembolism is a harbinger of occult cancer in patients with diverticular disease.

Keywords: epidemiology, pulmonary embolism, deep venous thrombosis, diverticulitis, perforated diverticulitis, cancer

\section{Introduction}

There is compelling evidence that venous thromboembolism (VTE), including pulmonary embolism (PE) and deep venous thrombosis (DVT), is a complication of cancer. $^{1,2}$ On the other hand, among patients diagnosed with VTE, studies have shown a two- to fourfold increased one-year risk of subsequent cancer compared with the general population. ${ }^{1,3,4}$ Thus, VTE may be considered a sign of an occult cancer. ${ }^{1}$ The pathogenetic mechanisms underlying this association are thought to include hypercoagulability due to activation of clotting by tumor cells, vessel wall injury, and stasis. ${ }^{1,5}$

Diverticular disease (DD), a common condition in the Western world, occurs by herniation of mucosa and submucosa through the muscular layer of the colonic wall. $^{6}$ In the western world, the prevalence of DD is reported to be up to $50 \%$ of 
adults aged 60 years and older. ${ }^{7}$ The condition remains asymptomatic in most cases; however, around five percent of patients develop complications such as bowel obstruction, inflammation, bleeding, or perforation. ${ }^{8}$ The most common complications include diverticular bleeding and diverticulitis. ${ }^{6}$ Compared with the general population, patients with both diverticulitis and perforated diverticulitis are suggested to have a $40 \%$ increased risk of developing VTE. ${ }^{9}$

Previous studies have shown a two- to threefold increased one-year risk of cancer after VTE among patients with concurrent liver disease, inflammatory bowel disease, rheumatoid arthritis, and diabetes. ${ }^{10-13}$ These studies have contributed with evidence that can be used for clinical decision-making when planning the diagnostic workup for occult cancer in patients with VTE and specific comorbidities. However, it remains unknown whether VTE could also be considered a marker of occult cancer in patients with DD. Thus, enhanced understanding of cancer risk after VTE in DD patients is needed.

We, therefore, conducted a population-based cohort study in Denmark to examine the risk of cancer following VTE among patients with DD and compared the observed risk of cancer with the expected based on national cancer incidence rates.

\section{Materials and Methods \\ Setting}

We conducted a population-based cohort study based on the entire Danish population during 1 April 1996-31 December 2017. We obtained prospectively collected data from the Danish National Patient Registry (DNPR) and the Danish Cancer Registry (DCR). Since 1968, all Danish residents are assigned a unique ten-digit civil registration number and registered in the Civil Registration System. This allows exact individual-level linkage between the DNPR and the DCR. ${ }^{14}$ All Danish residents are covered by the tax-financed public health insurance. ${ }^{15}$ The study was conducted in a setting without specific cancer screening for patients with DD; however, DD patients were invited to the Danish national colorectal cancer screening from 2014-2017 on equal conditions as all other Danes aged $50-74$ years. ${ }^{16}$

\section{Venous Thromboembolism and Diverticular Disease Cohort}

We searched the DNPR to identify all patients with a firsttime hospital-based diagnosis (primary [ie main diagnosis of hospital contact] or secondary [ie diagnoses recorded in addition to the primary diagnosis], excluding emergency room diagnoses) of VTE during 1996-2016. The inclusion ended on 31 December 2016 to ensure at least one year of follow-up after VTE for all included patients. VTE events included both PE and DVT (see Supplementary Table 1 for ICD codes). Since 1977, the DNPR has recorded information on all patients discharged from Danish nonpsychiatric hospitals. Since 1995, all psychiatric inpatients, psychiatric and somatic outpatients, and emergency room contacts have been included in the DNPR. ${ }^{17}$ The information recorded in the DNPR includes the civil registration number, dates of hospital admission and discharge, treatments, examinations, and up to 20 discharge diagnoses. Diagnoses are coded according to the International Classification of Diseases, Eighth revision (ICD-8) until 31 December 1993 and according to the Tenth revision (ICD-10) thereafter. ${ }^{17}$ Since 1996, surgical procedures have been coded according to the Nordic MedicoStatistical Committee (NOMESCO) classification of surgical procedures.

We restricted the VTE cohort to patients with a prior or concurrent diagnosis of DD (Figure 1 illustrates the study population). We applied two methods to further categorize patients with DD, to be able to investigate if the type and treatment of DD could have an impact on the risk of cancer after VTE. First, based on ICD-8 and ICD-10 codes, we categorized DD patients into diverticulitis and perforated diverticulitis (see Supplementary Table 1 for ICD codes). Second, based on ICD-10 and NOMESCO codes, we categorized DD patients into those surgically treated, conservatively treated, and others (see Supplementary Table 1 for ICD codes). The total number of DD patients with VTE were identified from all DD diagnoses codes (ICD-8, ICD-10) while subgroups were identified as described above. Thus, the total number of patients in each subgroup did not correspond to the total number of DD patients included in our study. The groups within the two different DD categorization methods were mutually exclusive. Hence, when multiple DD codes were recorded, patients were assigned to the DD group based on the diagnosis recorded closest to their VTE diagnosis.

\section{Cancer Outcomes}

We linked the cohort of VTE patients with DD to the DCR to identify incident cancers recorded after a VTE. The DCR has recorded all cases of cancer in the Danish population since 1978, coded according to ICD-10. ${ }^{18}$ Cancers 


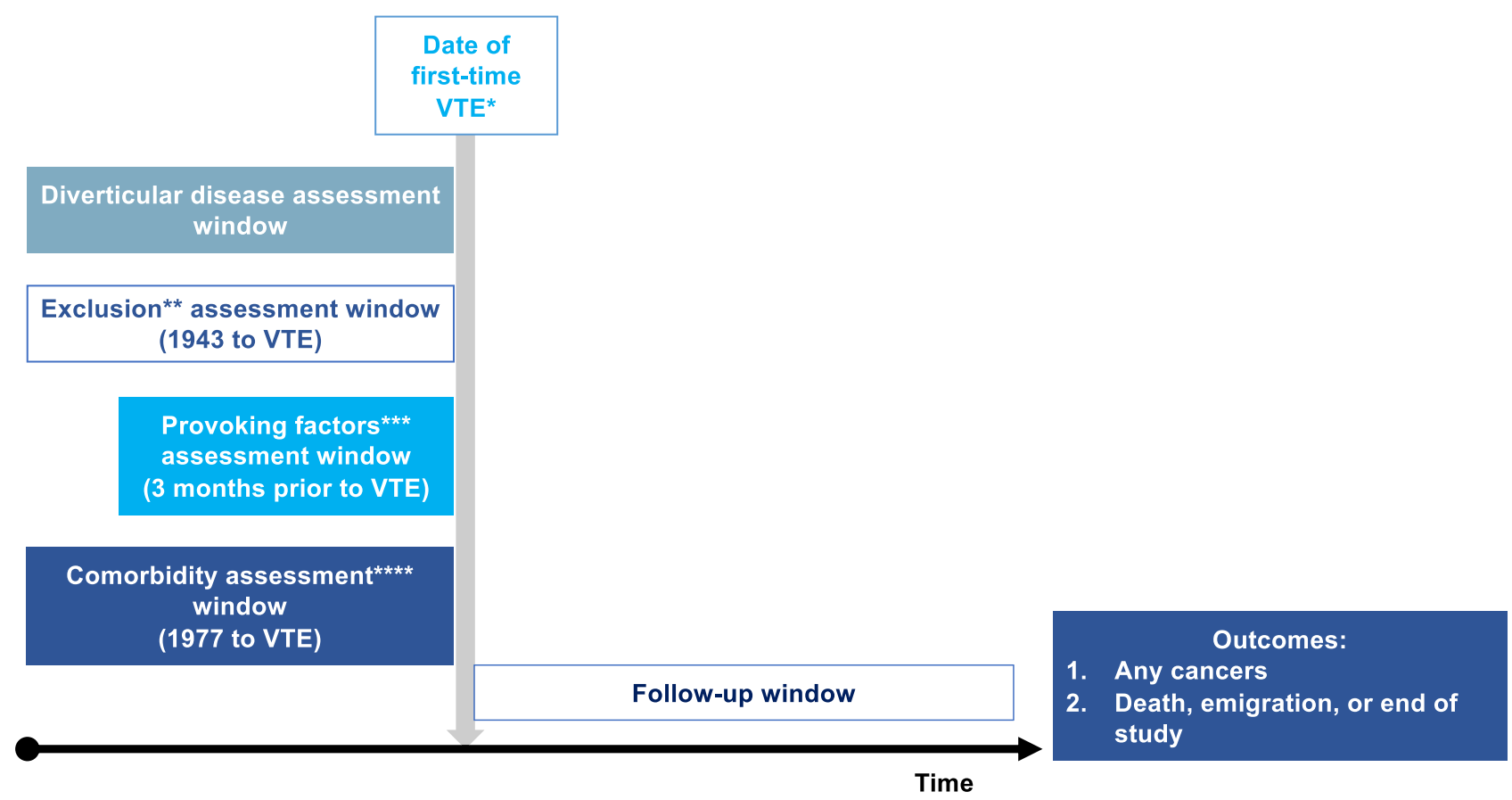

Figure I Study flow diagram (*Venous thromboembolism, **Any cancers, ***Fractures/trauma, pregnancy, or surgery, ****According to Charlson Comorbidity Index).

are categorized according to the annual cancer report published by The Danish Health Data Authority in $2018 .{ }^{19}$ Using data from the DCR, we excluded all patients with a cancer diagnosis recorded before or at the date of VTE diagnosis (Figure 1).

\section{Covariates}

We used the DNPR to ascertain the presence of previous provoking factors for VTE and comorbidities recorded before the date of VTE (Figure 1). We considered fracture/trauma, pregnancy, or surgery recorded in the DNPR within three months prior to the VTE event (admission date) as provoking factors for VTE. ${ }^{20}$ VTEs without these conditions recorded prior to diagnosis were considered as unprovoked. ${ }^{20}$ Based on hospital discharge diagnoses recorded in the DNPR from 1977 to the date of VTE, we used the Charlson Comorbidity Index (CCI) to measure the burden of comorbidity. The CCI is a scoring system that assigns from one to six points to a range of diseases based on their impact on mortality (see Supplementary Table 2 for ICD codes and assigned weighting). ${ }^{21}$ According to the calculated CCI score, we categorized all VTE patients with DD into three subgroups: low (no comorbidity) $=\mathrm{CCI}$ score of 0 , medium $=\mathrm{CCI}$ score of $1-2$, or high $=$ CCI score of 3 or more. Of note, we applied a modified CCI excluding any previous tumors before the VTE diagnosis from counting in the index, because any cancers recorded before the index date were excluded initially.

\section{Statistical Analysis}

We categorized all VTE patients with a history of DD according to age, sex, year of VTE diagnosis, type of $\mathrm{DD}$, type of treatment for DD, CCI score, and presence of provoking factors. We followed all VTE patients with DD from the date of VTE diagnosis until occurrence of a first-time cancer diagnosis, death, emigration, or administrative end of follow-up (31 December 2017), whichever occurred first. We divided the follow-up period into one year (first year) and more than one year (second and subsequent years) following the VTE diagnosis.

We calculated the absolute risks of cancer after VTE as the cumulative incidence proportions considering death as a competing risk. ${ }^{22}$ As a measure of the relative risk, we calculated standardized incidence ratios (SIRs) - the ratio of the observed number of cancers to the expected number of cancers. We used the national cancer incidence rates to calculate the expected number of cancer cases after a firsttime hospital-based diagnosis of VTE according to sex, age, and calendar period of diagnosis (one year intervals). Multiplying the number of years of follow-up by the incidence rates yielded the number of expected cancer cases if patients with VTE and DD had the same risk of cancer as the general population. We calculated $95 \%$ confidence intervals (CIs) for SIRs under the assumption that the observed number of cases in a specific category followed a Poisson 
distribution. ${ }^{22}$ When the observed number was less than ten, the exact 95\% CIs were used; otherwise Byar's approximation was used. ${ }^{22}$ Both SIRs and absolute risks were stratified by sex, age, calendar period, CCI score at the date of VTE, and presence of classic provoking factors for VTE recorded in the DNPR within three months prior to the VTE. We conducted analyses for VTE and for PE and DVT separately. Patients with a simultaneous diagnosis of PE and DVT were considered as having PE.

We conducted all statistical analyses using SAS statistical software package, V.9.4 (SAS Institute, Cary, North
Carolina, USA). The study was reported to the Danish Data Protection Agency by Aarhus University (record number 2016-051-000001/811). According to Danish legislation, no approval from an ethics committee or informed consent from patients are required for register-based studies. $^{23}$

\section{Results \\ Descriptive Data}

We followed 3406 incident VTE patients with a diagnosis of DD for a median follow-up time of 3.0 years

Table I Characteristics of Patients Having Venous Thromboembolism (VTE) and Diverticular Disease (DD). Denmark I996-2016

\begin{tabular}{|c|c|c|c|}
\hline Variable & Patients with DD and VTE & Patients with DD and PE & Patients with DD and DVT \\
\hline Total number & 3406 & $1696(49.8)$ & $1710(50.2)$ \\
\hline Median follow-up time (IQR), years & $3.0(1.0-6.0)$ & $2.1(0.3-4.7)$ & $3.9(1.7-7.2)$ \\
\hline Median age at VTE diagnosis, years & $77.2(68.4-83.7)$ & $77.8(69.7-83.9)$ & $76.5(66.7-90.7)$ \\
\hline $0-69$ & $981(28.8)$ & $437(12.8)$ & $544(16.0)$ \\
\hline $70-84$ & $1720(50.5)$ & $895(26.3)$ & $825(24.2)$ \\
\hline $85+$ & $705(20.7)$ & $364(10.7)$ & $34 I(10.0)$ \\
\hline \multicolumn{4}{|l|}{ Sex } \\
\hline Female & $2148(63.1)$ & $1056(31.0)$ & $1092(32.1)$ \\
\hline Male & $1258(36.9)$ & $640(18.8)$ & $618(18.1)$ \\
\hline \multicolumn{4}{|l|}{ Year of VTE diagnosis } \\
\hline $1996-1999$ & $293(8.6)$ & $110(3.2)$ & $183(5.4)$ \\
\hline $2000-2004$ & $590(17.3)$ & $260(7.6)$ & $330(9.7)$ \\
\hline 2005-2009 & $796(23.4)$ & $353(10.4)$ & $443(13.0)$ \\
\hline $2010-2014$ & II $57(34.0)$ & $608(17.9)$ & $549(16.1)$ \\
\hline $2015-2016$ & $570(16.7)$ & $365(10.7)$ & $205(6.0)$ \\
\hline \multicolumn{4}{|l|}{ Type of DD } \\
\hline Diverticulitis & $213(6.3)$ & $94(5.5)$ & $119(7.0)$ \\
\hline Perforated diverticulitis & $300(8.8)$ & $162(9.6)$ & $138(8.1)$ \\
\hline \multicolumn{4}{|l|}{ Type of treatment ${ }^{\mathrm{b}}$} \\
\hline DD surgically treated & $50(1.7)$ & $22(0.7)$ & $28(0.9)$ \\
\hline DD conservatively treated & $2402(81.0)$ & I $322(44.6)$ & $1080(36.4)$ \\
\hline Other cases of diverticular disease & $512(17.3)$ & $164(5.5)$ & $348(11.7)$ \\
\hline \multicolumn{4}{|l|}{ Charlson Comorbidity Index $(\mathrm{CCl})$} \\
\hline CCl: Low & $1227(36.0)$ & $549(16.1)$ & $678(19.9)$ \\
\hline CCl: Medium & $150 \mid(44.1)$ & $75 I(22.1)$ & $750(22.0)$ \\
\hline CCl: High & $678(19.9)$ & $396(11.6)$ & $282(8.3)$ \\
\hline \multicolumn{4}{|l|}{ Provoking factor ${ }^{c}$} \\
\hline Absent & $2570(75.5)$ & $1264(37.1)$ & $1306(38.3)$ \\
\hline Present & $836(24.5)$ & $432(12.7)$ & $404(11.8)$ \\
\hline
\end{tabular}

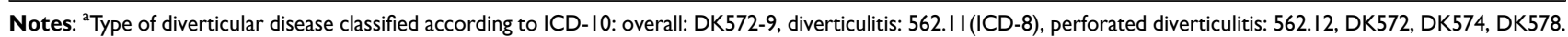
${ }^{\text {b}}$ Type of DD treatment classified according to ICD- I0: Surgically treated: DK572-9 and KJF, KJG, or KJAH0I, conservatively treated: inpatient diagnoses of DK572-9, other: outpatient diagnoses of DK572-9. 'VTE provoking factors were classified as fracture/trauma, pregnancy, or surgery recorded in the DNPR within three months prior to the VTE event (admission date). Patients without these were considered to have no previous presence of provoking factors.

Abbreviations: PE, pulmonary embolism; DVT, deep venous thrombosis; IQR, interquartile rage. 
(interquartile range [IQR]: 1.0-6.0). Table 1 shows patient characteristics. The median age at VTE diagnosis was 77.2 years (IQR: 68.4-83.7), and more than half of the patients were female (63.1\%). Overall, 836 patients $(24.5 \%)$ had a provoking factor diagnosed within 90 days prior to the VTE, and 1501 (44.1\%) had a medium CCI score. Among included VTE patients with DD, 1696 (49.8\%) had PE (Table 1). The characteristics at date of VTE were virtually equal across patients with $\mathrm{PE}$ and patients with DVT.

\section{First Year of Follow-Up}

Within one year after VTE diagnosis, 212 patients were diagnosed with cancer. The corresponding one-year absolute risk was $6.2 \%$ (CI: $5.5-7.1$ ) (Figure 2 and Table 2). The total number of expected cancer cases during the first year was 72.7, yielding a SIR of 2.9 (95\% CI: 2.5-3.3) (Table 3). The SIR was similar between men and women, across different age groups, calendar periods, CCI scores, and presence of provoking factors. In contrast, the SIR was higher among patients diagnosed with PE (SIR $=3.6$ [95\% CI: 3.0-4.3]) than among patients with DVT (SIR $=2.3$ [95\% CI: 1.8-2.8]). SIRs were particularly elevated during the first year of follow-up for cancers of the stomach (SIR $=9.2$ [95\% CI: 4.2-17.4]), pancreas (SIR $=6.8$ [95\% CI: 3.5-12.0]), the ovary (SIR = 11.5 [95\% CI: $5.7-20.5]$ ), and the kidney (SIR=8.2 [95\% CI: 3.6-16.2]) (Table 4).

\section{Second and Subsequent Years of Follow- Up}

During the second and subsequent years, a total of 337 cancers diagnoses were observed (Table 3). Overall, the SIR decreased to 1.1 (95\% CI: 1.0-1.3). SIRs had point estimates around 1.0 across all stratifications of patient characteristics and cancer sites.

\section{Type and Treatment of Diverticular Disease}

We followed 213 (6.2\%) VTE patients with a history of diverticulitis and $300(8.8 \%)$ with perforated diverticulitis (Table 1). According to type of treatment, 50 $(1.7 \%)$ were surgically treated, $2402(81.0 \%)$ were conservatively treated, and $512(17.3 \%)$ were classified as

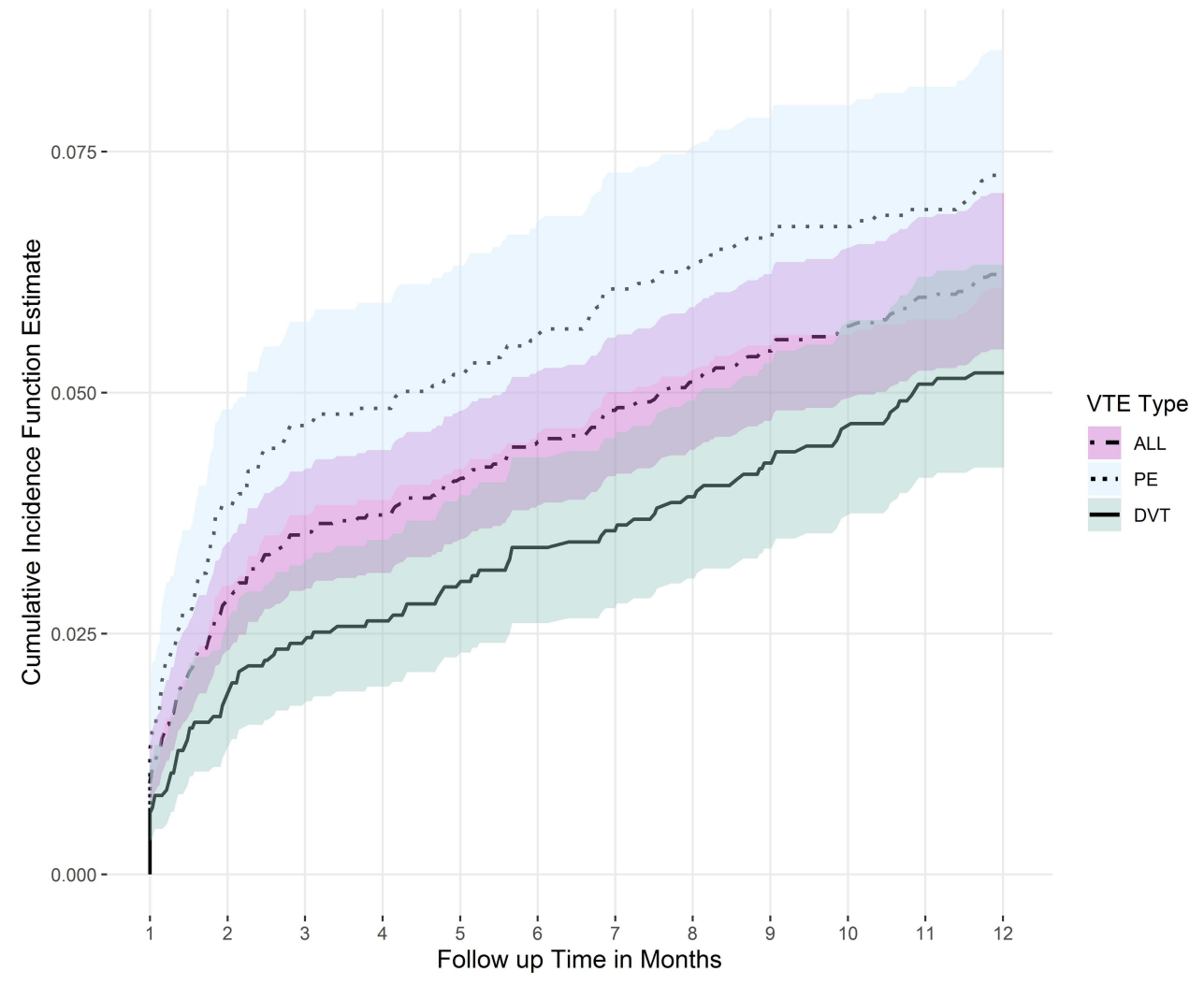

Figure 2 Cumulative incidence proportion of cancer patients with diverticular disease and venous thromboembolism. 
Table 2 One-Year Absolute Risks of Cancer and 95\% Confidence Intervals (Cl's) Among Patients with Venous Thromboembolism (VTE) and Diverticular Disease (DD). Denmark 1996-2017

\begin{tabular}{|c|c|}
\hline Variable & $\begin{array}{l}\text { Absolute First Year-Risk } \\
\qquad(95 \% \mathrm{Cl})\end{array}$ \\
\hline All & $6.2(5.5-7.1)$ \\
\hline \multicolumn{2}{|l|}{ Median age at VTE diagnosis, years } \\
\hline $0-69$ & $4.9(3.7-6.4)$ \\
\hline $70-84$ & $7.7(6.5-9.0)$ \\
\hline $85+$ & $4.5(3.2-6.3)$ \\
\hline \multicolumn{2}{|l|}{ Sex } \\
\hline Female & $5.5(4.5-6.5)$ \\
\hline Male & $7.6(6.2-9.1)$ \\
\hline \multicolumn{2}{|l|}{ Year of VTE diagnosis } \\
\hline $1996-1999$ & $5.5(3.3-8.5)$ \\
\hline $2000-2004$ & $4.6(3.1-6.5)$ \\
\hline 2005-2009 & $6.3(4.7-8.1)$ \\
\hline $2010-2014$ & $6.6(5.2-8.1)$ \\
\hline $2015-2016$ & $7.5(5.6-9.9)$ \\
\hline \multicolumn{2}{|l|}{ VTE Type ${ }^{a}$} \\
\hline PE & $7.3(6.1-8.6)$ \\
\hline DVT & $5.2(4.2-6.3)$ \\
\hline \multicolumn{2}{|l|}{ Charlson Comorbidity Index (CCl) } \\
\hline CCl: Low & $6.8(5.5-8.3)$ \\
\hline CCl: Medium & $5.8(4.7-7.1)$ \\
\hline CCl: High & $6.2(4.6-8.2)$ \\
\hline \multicolumn{2}{|l|}{ Provoking factors ${ }^{\mathrm{b}}$} \\
\hline Absent & $6.5(5.6-7.5)$ \\
\hline Present & $5.3(3.9-6.9)$ \\
\hline
\end{tabular}

Notes: aICD-I0: pulmonary embolism (PE) DI26, deep venous thromboembolism (DVT) DI80.I-3. 'bTE provoking factors were classified as fracture/trauma, pregnancy, or surgery recorded in the Danish National Patient Registry (DNPR) within three months prior to the VTE event (admission date). Patients without these were considered to have no previous presence of provoking factors.

"other". During the first year of follow-up, VTE patients with a history of perforated diverticulitis had a higher SIR for cancer $(\operatorname{SIR}=5.3[95 \%$ CI: 3.5-7.7]) than patients with a history of diverticulitis $(\mathrm{SIR}=3.8$ [95\% CI: 2.2-6.3]) (Table 5), although 95\% CIs were overlapping. Concerning type of treatment, conservatively treated DD patients had the highest SIR of cancer $(\mathrm{SIR}=3.2$ [95\% CI: 2.8-3.8]). In the second and subsequent years of follow-up, the SIR decreased in both patients with diverticulitis (SIR $=1.1 \quad[95 \% \mathrm{CI}$ : $0.7-$ 1.6]) and perforated diverticulitis (SIR $=1.3[95 \% \mathrm{CI}$ :
0.9-1.9]). Also, the SIR in the group of conservatively treated DD patients decreased $(\mathrm{SIR}=1.1[95 \% \mathrm{CI}$ : $0.9-1.2])$.

\section{Discussion Key Results}

In this population-based cohort study of patients with both VTE and DD, the one-year absolute risk of cancer was six percent. Compared with the general population, the oneyear risk of cancer after the VTE diagnosis was increased three-fold. Relative risks of cancer were particularly elevated for cancers of the stomach, pancreas, ovary, and kidney. One-year absolute and relative risks of cancer were higher among VTE patients diagnosed with perforated diverticulitis than among patients diagnosed with diverticulitis.

\section{Interpretation}

Although DD is suggested as a risk factor for VTE, our study is the first to investigate the risk of cancer following VTE in patients with DD. ${ }^{9}$ Our finding of an increased risk of cancer subsequent to VTE in DD patients is in line with previous findings among patients hospitalized with VTE in the general population and indicates that VTE in patients with DD should be regarded as much a harbinger of occult cancer as it is for VTEs in the general population. ${ }^{1,3,4}$ Generally, cancer diagnosed within one year after VTE tends to be associated with an advanced stage of cancer and a poor prognosis. ${ }^{24}$ Screening for prevalent malignancy following VTE leads to an early detection of occult cancer at an earlier cancer stage, which may be associated with improved treatment. ${ }^{25,26}$ However, cancer screening is generally not recommended after VTE.

The increased short-termrisk of cancer after VTE may be explained by heightened diagnostic efforts among patients with VTE or DD. ${ }^{27}$ Figure 2 is depicting the cumulative incidence proportion of cancer within the first year after VTE in the DD population. The curve is particularly steep during the first month. This could be explained by a well-known increased risk of cancer after VTE and/or by a higher degree of examinations for potential occult cancer (called detection bias). However, if detection bias had occurred, the period of increased prevalent cancer diagnosis during the first year of follow-up would have been followed by a compensatory 
Table 3 Age, Sex, and Calendar Period Standardized Incidence Ratios (SIRs) of Cancer and 95\% Confidence Intervals (Cl's) Among Patients with Diverticular Disease (DD) and Venous Thromboembolism (VTE). Denmark 1996-2017

\begin{tabular}{|c|c|c|c|c|}
\hline \multirow[t]{2}{*}{ Variable } & \multicolumn{2}{|c|}{ First Year After VTE } & \multicolumn{2}{|c|}{$>$ I Year After VTE } \\
\hline & O/E & SIR (95\% CI) & O/E & SIR $(95 \% \mathrm{CI})$ \\
\hline Total & $212 / 72.7$ & $2.9(2.5-3.3)$ & $337 / 300.7$ & I.I (I.0-1.3) \\
\hline \multicolumn{5}{|l|}{ Sex } \\
\hline Female & || $7 / 4 \mid .5$ & $2.8(2.3-3.4)$ & $199 / 176.0$ & I.I (I.0-I.3) \\
\hline Male & $95 / 31.2$ & $3.0(2.5-3.7)$ & $138 / 124.6$ & I.I (0.9-I.3) \\
\hline \multicolumn{5}{|c|}{ Age at having VTE diagnosis } \\
\hline $0-69$ years & $48 / 14.2$ & $3.4(2.5-4.5)$ & $120 / 100.6$ & $1.2(1.0-1.4)$ \\
\hline $70-84$ years & $132 / 43.0$ & $3.1(2.6-3.6)$ & $178 / 163.2$ & I.I (0.9-I.3) \\
\hline $85+$ years & $32 / 15.4$ & $2.1(1.4-2.9)$ & $39 / 36.8$ & I.I (0.8-I.5) \\
\hline \multicolumn{5}{|c|}{ Year of VTE diagnosis } \\
\hline $1996-1999$ & $16 / 4.9$ & $3.3(1.9-5.3)$ & $33 / 36.3$ & $0.9(0.6-1.3)$ \\
\hline 2000-2004 & $27 / 10.6$ & $2.5(1.7-3.7)$ & $96 / 74.1$ & $1.3(1.1-1.6)$ \\
\hline $2005-2009$ & $50 / 16.7$ & $3.0(2.2-4.0)$ & $99 / 93.5$ & I.I (0.9-I.3) \\
\hline $2010-2014$ & $76 / 27.2$ & $2.8(2.2-3.5)$ & $97 / 85.1$ & I.I (0.9-I.4) \\
\hline $2015-2016$ & $43 / 13.3$ & $3.2(2.3-4.4)$ & $12 / 11.6$ & $1.0(0.5-1.8)$ \\
\hline \multicolumn{5}{|l|}{ Type of VTE ${ }^{a}$} \\
\hline PE & $123 / 33.9$ & $3.6(3.0-4.3)$ & $126 / 114.0$ & I.I (0.9-I.3) \\
\hline DVT & $89 / 38.8$ & $2.3(1.8-2.8)$ & $221 / 186.7$ & I.I (I.0-I.3) \\
\hline \multicolumn{5}{|c|}{ Charlson Comorbidity Index } \\
\hline CCI: Low & $83 / 25.6$ & $3.2(2.6-4.0)$ & $150 / 136.4$ & I.I (0.9-I.3) \\
\hline CCl: Medium & $87 / 33.1$ & $2.6(2.1-3.2)$ & $132 / 130.1$ & $1.0(0.9-1.2)$ \\
\hline CCl: High & $42 / 14.0$ & $3.0(2.2-4.1)$ & $55 / 34.1$ & $1.6(1.2-2.1)$ \\
\hline \multicolumn{5}{|c|}{ Provoking factor ${ }^{b}$} \\
\hline Absent & $168 / 56 . \mid$ & $3.0(2.6-3.5)$ & $265 / 231.0$ & I.I (I.0-1.3) \\
\hline Present & $44 / 16.5$ & $2.7(1.9-3.6)$ & $72 / 69.7$ & $1.0(0.8-1.3)$ \\
\hline
\end{tabular}

Notes: aCD-10: pulmonary embolism (PE) DI26, deep venous thromboembolism (DVT) DI80.I-3. ' VTE provoking factors were classified as fracture/trauma, pregnancy, or surgery recorded in the Danish National Patient Registry (DNPR) within three months prior to the VTE event (admission date). Patients without these were considered to have no previous presence of provoking factors.

Abbreviations: $\mathrm{CCl}$, Charlson Comorbidity Index; O, observed; E, expected.

decrease in the following follow-up period. ${ }^{1,27}$ We did not see such a pattern.

The analysis concerning type of DD showed an increased risk of cancer following VTE independently of type of DD and type of treatment. Especially, patients diagnosed with perforated DD had a high one-year absolute and relative risk of cancer. Although our analyses were unable to explore the underlying mechanism, it is likely that immobilization, disease activity, and surgical treatment may play a role in this particularly high risk. $^{28}$

Relative risks of cancer were particularly elevated for cancers of the stomach, pancreas, ovary, and kidney. This was in line with previous studies that found a two- to threefold increased one-year risk of cancer after VTE. ${ }^{10-13}$

\section{Limitations}

Strengths of the current study includes the populationbased design in a setting with free access to healthcare and the high quality and continuously updated data on VTE, comorbidities, and cancer diagnoses. Further, the Danish national registries allowed us to study the entire population for a long period of time with complete followup minimizing the potential for selection and referral bias. The DNPR contains hospital diagnoses only and we did not have accessible data on diagnoses made by general practitioners. Fortunately, the majority of patients suspected for a VTE would be referred to a hospital department for further diagnosis and treatment by their general practitioner. Hence, they will be captured in the DNPR and included in our study. 
Table 4 Age, Sex, and Calendar Period Standardized Incidence Ratios (SIRs) of Cancer and 95\% Confidence Intervals (Cl's) Among Patients with Venous Thromboembolism (VTE) and Diverticular Disease. Denmark 1996-2017. Numbers and SIRs Below Five are Marked with $<5$ to Secure Anonymity According to Danish Legislation

\begin{tabular}{|c|c|c|c|c|}
\hline \multirow[t]{2}{*}{ Cancer Groups } & \multicolumn{2}{|c|}{ First Year After VTE } & \multicolumn{2}{|c|}{$>$ I Year After VTE } \\
\hline & O/E & SIR $(95 \% \mathrm{CI})$ & O/E & $\operatorname{SIR}(95 \% \mathrm{CI})$ \\
\hline All & $212 / 72.7$ & $2.9(2.5-3.3)$ & $337 / 300$ & I.I (I.0-1.3) \\
\hline Stomach & $9 / 1.0$ & $9.2(4.2-17.4)$ & $6 / 3.9$ & $1.5(0.6-3.3)$ \\
\hline Large intestine incl. Colon rectosigmoid & $24 / 6.1$ & $3.9(2.5-5.9)$ & $35 / 24.9$ & $1.4(1.0-2.0)$ \\
\hline Rectum & $<5$ & NA & $9 / 10.3$ & $0.9(0.4-1.7)$ \\
\hline Pancreas & $12 / 1.8$ & $6.8(3.5-12.0)$ & $9 / 7.2$ & $1.3(0.6-2.4)$ \\
\hline Lung, bronchi and trachea & $25 / 7.1$ & $3.5(2.3-5.2)$ & $48 / 28.2$ & $1.7(1.3-2.3)$ \\
\hline Malignant melanoma & $<5$ & NA & $7 / 7.3$ & $1.0(0.4-2.0)$ \\
\hline Other skin cancer (excl. Basal cell carcinoma) & $5 / 4.4$ & I.I (0.4-2.7) & $33 / 20.5$ & $1.6(1.1-2.3)$ \\
\hline Breast & $7 / 6.8$ & $1.0(0.4-2.1)$ & 19/28.1 & $0.7(0.4-1.1)$ \\
\hline Uterus & $<5$ & NA & $7 / 5.7$ & $1.2(0.5-2.5)$ \\
\hline Ovary & $\mathrm{II} / \mathrm{I} .0$ & I I.5 (5.7-20.5) & $<5$ & NA \\
\hline Prostate & $21 / 6.1$ & $3.5(2.1-5.3)$ & $22 / 24.1$ & $0.9(0.6-1.4)$ \\
\hline Kidney & $8 / 1.0$ & $8.2(3.6-16.2)$ & $<5$ & NA \\
\hline Urinary bladder & $7 / 3.3$ & $2.1(0.8-4.3)$ & $12 / 13.2$ & $0.9(0.5-1.6)$ \\
\hline Non-Hodgkin malignant lymphoma & $10 / 2.4$ & $4.2(2.0-7.7)$ & $7 / 9.9$ & $0.7(0.3-1.5)$ \\
\hline Metastases and non-specified cancer in lymph nodes & $20 / 1.6$ & $12.8(7.8-19.8)$ & $5 / 6.2$ & $0.8(0.3-1.9)$ \\
\hline Basal cell carcinoma & $16 / 15.8$ & $1.0(0.6-1.6)$ & $71 / 68.1$ & $1.0(0.8-1.3)$ \\
\hline
\end{tabular}

Abbreviations: O, observed; E, expected.

Table 5 One-Year Absolute Risks and Age, Sex, and Calendar Period Standardized Incidence Ratios (SIRs) of Cancer and 95\% Confidence Intervals (Cl's) Among Patients with Venous Thromboembolism (VTE) and Diverticular Disease (DD) According to Type and Treatment of DD. Denmark 1996-2017. Numbers and SIRs Below Five are Marked with <5 to Secure Anonymity According to Danish Legislation

\begin{tabular}{|c|c|c|c|c|c|}
\hline \multirow[t]{2}{*}{ Variable } & \multirow[t]{2}{*}{ Absolute First Year-Risk $(95 \% \mathrm{Cl})$} & \multicolumn{2}{|c|}{ First Year After VTE } & \multicolumn{2}{|c|}{ >I Year After VTE } \\
\hline & & O/E & SIR (95\% CI) & O/E & $\operatorname{SIR}(95 \% \mathrm{CI})$ \\
\hline \multicolumn{6}{|l|}{ Type of DD ${ }^{a}$} \\
\hline Diverticulitis & $7.5(4.5-11.6)$ & $16 / 4.2$ & $3.8(2.2-6.3)$ & $27 / 24.1$ & I.I $(0.7-1.6)$ \\
\hline Perforated diverticulitis & $9.3(6.4-13.0)$ & $28 / 5.3$ & $5.3(3.5-7.7)$ & $32 / 24.3$ & $1.3(0.9-1.9)$ \\
\hline \multicolumn{6}{|l|}{ Type of treatment ${ }^{b}$} \\
\hline DD surgically treated & $2.0(0.2-9.4)$ & $<5$ & NA & $9 / 5.6$ & $1.6(0.7-3.1)$ \\
\hline DD conservatively treated & $6.8(5.8-7.8)$ & $163 / 50.5$ & $3.2(2.8-3.8)$ & $210 / 197.8$ & I.I (0.9-I.2) \\
\hline Other cases of DD & $4.1(2.6-6.1)$ & $21 / 12.5$ & $1.7(1.0-2.6)$ & $59 / 48.2$ & $1.2(0.9-1.6)$ \\
\hline
\end{tabular}

Notes: ${ }^{2}$ Type of diverticular disease classified according to ICD-10: overall: DK572-9, diverticulitis: 562.1 I (ICD-8), perforated diverticulitis: 562. 12, DK572, DK574, DK578. ${ }^{\mathrm{b}}$ Type of diverticular disease treatment classified according to ICD-I0: Surgically treated: DK572-9 and KJF, KJG, or KJAH0I, conservatively treated: inpatient diagnoses of DK572-9, other: Outpatient diagnoses of DK572-9. 
Limitations of our study include the potential misclassification of diagnostic coding of VTE, DD, cancer, and other comorbidities. However, the positive predictive value has been found to be sufficiently high for both VTE (75-90\%), ${ }^{29} \mathrm{DD}$ $(98 \%),{ }^{30}$ cancer $(95-98 \%),{ }^{31}$ and other comorbidities $(98 \%){ }^{21}$ To circumvent some potential misclassification of the VTE diagnosis, we excluded patients diagnosed in emergency departments, because these often are based only on clinical suspicion. ${ }^{29}$ Further, the diagnostic coding of DD in the DNPR does not differentiate between patients with diverticulosis and diverticulitis as well as between patients with uncomplicated diverticulosis or with specific diverticular complications. ${ }^{30}$ Instead, we combined subgroups of diagnostic codes to categorize the type of DD to investigate the risk of cancer following VTE in specific groups of DD patients. Patients conservatively treated had a higher cancer incidence rate, than patients surgically treated. Surgical resection of an inflamed bowel segment may secure a lower degree of intestinal and systemic inflammation, in turn decreasing the risk for subsequent cancer development. The cohort includes all patients with a history of prior DD, irrespective of treatment status. Therefore, some patients underwent surgery and might no longer have active DD at the time of VTE diagnosis. This detail should be kept in mind when interpreting our findings. Lastly, it should be noted that the grouping of treatment for DD was conducted on other codes than type of DD. Hence, it is not possible to directly compare the estimates showed for different treatment of DD (ie surgical, conservative, or other) and different type of DD (ie diverticulitis or perforated diverticulitis).

\section{Conclusion}

In conclusion, compared with the general population, VTE patients with DD had a three-fold increased risk of cancer within the first year following VTE. Our results suggest that VTE may be a harbinger of occult cancer in patients with DD.

\section{Acknowledgments}

The Department of Clinical Epidemiology is involved in studies with funding from various companies in the form of research grants to and administered by Aarhus University. None of these studies are related to the present study.

\section{Disclosure}

The authors report no conflicts of interest in this work.

\section{References}

1. Sorensen HT, Mellemkjaer L, Steffensen FH, Olsen JH, Nielsen GL. The risk of a diagnosis of cancer after primary deep venous thrombosis or pulmonary embolism. $N$ Engl J Med. 1998;338(17):11691173. doi:10.1056/NEJM199804233381701

2. Mulder FI, Horvath-Puho E, van Es N, et al. Venous thromboembolism in cancer patients: a population-based cohort study. Blood. 2021;137(14):1959-1969. doi:10.1182/blood.2020007338

3. Sorensen HT, Svaerke C, Farkas DK, et al. Superficial and deep venous thrombosis, pulmonary embolism and subsequent risk of cancer. Eur $J$ Cancer. 2012;48(4):586-593. doi:10.1016/j. ejca.2011.10.032

4. Murchison JT, Wylie L, Stockton DL. Excess risk of cancer in patients with primary venous thromboembolism: a national, population-based cohort study. Br J Cancer. 2004;91(1):92-95. doi:10.1038/ sj.bjc.6601964

5. Piccioli A, Prandoni P, Ewenstein BM, Goldhaber SZ. Cancer and venous thromboembolism. Am Heart J. 1996;132(4):850-855. doi:10.1016/s0002-8703(96)90321-x

6. Delvaux M. Diverticular disease of the colon in Europe: epidemiology, impact on citizen health and prevention. Aliment Pharmacol Ther. 2003;18(Suppl 3):71-74. doi:10.1046/j.09530673.2003.01720.x

7. Feuerstein JD, Falchuk KR. Diverticulosis and diverticulitis. J Pract Nurs. 2008;58(3):15-18. Fall.

8. Shahedi K, Fuller G, Bolus R, et al. Long-term risk of acute diverticulitis among patients with incidental diverticulosis found during colonoscopy. Clin Gastroenterol Hepatol. 2013;11(12):1609-1613. doi:10.1016/j.cgh.2013.06.020

9. Strate LL, Erichsen R, Horvath-Puho E, Pedersen L, Baron JA, Sorensen HT. Diverticular disease is associated with increased risk of subsequent arterial and venous thromboembolic events. Clin Gastroenterol Hepatol. 2014;12(10):1695-701 e1. doi:10.1016/j. cgh.2013.11.026

10. Montomoli J, Erichsen R, Sogaard KK, Kormendine Farkas D, Bloch Munster AM, Sorensen HT. Venous thromboembolism and subsequent risk of cancer in patients with liver disease: a population-based cohort study. BMJ Open Gastroenterol. 2015;2(1):e00043. doi:10.1136/bmjgast-2015-000043

11. Sorensen GV, Erichsen R, Svaerke C, Farkas DK, Sorensen HT. Risk of cancer in patients with inflammatory bowel disease and venous thromboembolism: a nationwide cohort study. Inflamm Bowel Dis. 2012;18(10):1859-1863. doi:10.1002/ibd.22870

12. Pedersen AB, Vandenbroucke J, Horvath-Puho E, Sorensen HT Venous thromboembolism and risk of cancer in patients with rheumatoid arthritis. J Thromb Haemost. 2017;15(12):2325-2332. doi: $10.1111 /$ jth. 13846

13. Christensen DH, Horvath-Puho E, Thomsen RW, et al. Venous thromboembolism and risk of cancer in patients with diabetes mellitus. $J$ Diabetes Complications. 2016;30(4):603-607. doi:10.1016/j. jdiacomp.2016.01.011

14. Schmidt M, Pedersen L, Sorensen HT. The Danish civil registration system as a tool in epidemiology. Eur J Epidemiol. 2014;29(8):541549. doi:10.1007/s10654-014-9930-3

15. Schmidt M, Schmidt SAJ, Adelborg K, et al. The Danish health care system and epidemiological research: from health care contacts to database records. Clin Epidemiol. 2019;11:563-591. doi:10.2147/ CLEP.S179083

16. Andersen JC. Nationale danske Guidelines for Behandling af divertikelsygdom: Udarbejdet af en arbejdsgruppe under Dansk Kirurgisk Selskab, maj 2011. Dansk Medicinsk Selskab; 2012.

17. Schmidt M, Schmidt SA, Sandegaard JL, Ehrenstein V, Pedersen L, Sorensen HT. The Danish national patient registry: a review of content, data quality, and research potential. Clin Epidemiol. 2015;7:449-490. doi:10.2147/CLEP.S91125 
18. Gjerstorff ML. The Danish cancer registry. Scand J Public Health. 2011;39(7 Suppl):42-45. doi:10.1177/1403494810393562

19. Sundhedsdatastyrelsen. Danish National board of health - annual cancer report; 2018. Available from: https://sundhedsdatastyrelsen. $\mathrm{dk} / \mathrm{da} /$ tal-og-analyser/analyser-og-rapporter/sygdomme/kraeft-_-nye tilfaelde. Accessed June 21, 2020.

20. Glynn RJ, Rosner B. Comparison of risk factors for the competing risks of coronary heart disease, stroke, and venous thromboembolism. Am J Epidemiol. 2005;162(10):975-982. doi:10.1093/aje/kwi309

21. Thygesen SK, Christiansen CF, Christensen S, Lash TL, Sorensen HT. The predictive value of ICD-10 diagnostic coding used to assess Charlson comorbidity index conditions in the population-based Danish national registry of patients. BMC Med Res Methodol. 2011;11:83. doi:10.1186/1471-2288-11-83

22. Breslow NE, Day NE. Statistical methods in cancer research. volume II-the design and analysis of cohort studies. IARC Sci Publ. 1987;82:1-406.

23. Ludvigsson JF, Haberg SE, Knudsen GP, et al. Ethical aspects of registry-based research in the Nordic countries. Clin Epidemiol. 2015;7:491-508. doi:10.2147/CLEP.S90589

24. Sorensen HT, Mellemkjaer L, Olsen JH, Baron JA. Prognosis of cancers associated with venous thromboembolism. $N$ Engl $J$ Med. 2000;343(25):1846-1850. doi:10.1056/NEJM200012213432504

25. Van Doormaal FF, Terpstra W, Van Der Griend R, et al. Is extensive screening for cancer in idiopathic venous thromboembolism warranted? J Thromb Haemost. 2011;9(1):79-84. doi:10.1111/j.15387836.2010.04101.x
26. Piccioli A, Lensing AW, Prins MH, et al. Extensive screening for occult malignant disease in idiopathic venous thromboembolism: a prospective randomized clinical trial. J Thromb Haemost. 2004;2 (6):884-889. doi:10.1111/j.1538-7836.2004.00720.x

27. Baron JA, Gridley G, Weiderpass E, Nyren O, Linet M. Venous thromboembolism and cancer. Lancet. 1998;351(9109):1077-1080. doi:10.1016/S0140-6736(97)10018-6

28. Strate LL. Lifestyle factors and the course of diverticular disease. Dig Dis. 2012;30(1):35-45. doi:10.1159/000335707

29. Severinsen MT, Kristensen SR, Overvad K, Dethlefsen C, Tjonneland A, Johnsen SP. Venous thromboembolism discharge diagnoses in the Danish national patient registry should be used with caution. J Clin Epidemiol. 2010;63(2):223-228. doi:10.1016/j. jclinepi.2009.03.018

30. Erichsen R, Strate L, Sorensen HT, Baron JA. Positive predictive values of the international classification of disease, 10th edition diagnoses codes for diverticular disease in the Danish national registry of patients. Clin Exp Gastroenterol. 2010;3:139-142. doi:10.2147/CEG.S13293

31. Storm HH, Michelsen EV, Clemmensen IH, Pihl J. The Danish cancer registry-history, content, quality and use. Dan Med Bull. 1997;44(5):535-539.
Clinical Epidemiology

\section{Publish your work in this journal}

Clinical Epidemiology is an international, peer-reviewed, open access, online journal focusing on disease and drug epidemiology, identification of risk factors and screening procedures to develop optimal preventative initiatives and programs. Specific topics include: diagnosis, prognosis, treatment, screening, prevention, risk factor modification,

Submit your manuscript here: https://www.dovepress.com/clinical-epidemiology-journal

\section{Dovepress}

systematic reviews, risk \& safety of medical interventions, epidemiology \& biostatistical methods, and evaluation of guidelines, translational medicine, health policies \& economic evaluations. The manuscript management system is completely online and includes a very quick and fair peer-review system, which is all easy to use. 